\title{
Approches probabilistes de la prédétermination des crues de la normale à l'extrême
}

\author{
Probabilistic approaches of flood predetermination
}

par Ph. Bois

Enshmg, Lthe, Inpg, Cnrs

Since it is not possible to determine in a reasonable manner the value of the peak flood of a river, only a probabilistic approach may provide satisfactory results. Such an approach only has a meaning in a decision-making framework to design for example hydraulic facilities. Hence, to talk about a flood with a 1000 year return period does not mean that one makes the hypothesis that the hydrological conditions remain stable in the 1000 years to come, but it is the flood that has a probability of one in thousand of occuring in a given year.

These probabistic methods are based on historical flood data for the oldest, and on floods and precipitations for the more recent. The associated problems of risk and sampling are presented by means of examples. The GRADEX method allows to evaluate, under certain hypotheses, the floods with long return periods and the AGREGEE method relates that method to floods with shorter return periods.

\section{I $\square$ LA PRÉDÉTERMINATION DES CRUES : UN OUTIL D'AIDE À LA DÉCISION}

La prédétermination des crues d'un bassin consiste à fournir une fonction de répartition vraisemblable des crues extrêmes, par exemple, la fonction de répartition (probabilité au non-dépassement) des pointes de crues maximales annuelles, d'une fréquence courante ( $10 \%$ de chance d'être dépassée une année quelconque), jusqu'à une fréquence rare, une chance sur 100 ou 1000 ou plus d'être dépassée.

Comme cette fonction de répartition $\mathrm{F}(X)=$ Probabilité $(x<X)$ concerne en général des maxima annuels, on a pris l'habitude de l'exprimer en Temps de Retour, avec : $\mathrm{T}(X)=1 /(1-\mathrm{F}(X))$; c'est ainsi qu'une crue de fréquence au non-dépassement 0,99 sera appelée crue centennale. Mais cela ne veut pas dire que l'on a une crue centennale par siècle ou que nous faisons l'hypothèse que le contexte hydrologique ne changera pas dans les siècles à venir, pour des raisons humaines ou climatiques. C'est une valeur numérique que l'on utilise pour dimensionner au mieux des ouvrages (digues, évacuateurs de crues, etc.), en fournissant au concepteur des valeurs avec des probabilités au non-dépassement pour une année quelconque et ceci pour les années à venir, à l'échelle humaine de la vie de l'ouvrage.

L'exemple suivant illustre cette problématique ; il s'agissait de protéger un bassin vénézuélien agricole contre les crues. L'hydrologue fournit les résultats suivants sur les maxima annuels de débits :

\begin{tabular}{|l|c|c|c|c|c|c|}
\hline $\mathbf{F}(\mathbf{Q})$ & 0,900 & 0,950 & 0,980 & 0,990 & 0,995 & 0,998 \\
\hline $\mathbf{Q}$ en m $\mathbf{3} / \mathbf{s}^{3}$ & 500 & 550 & 640 & 720 & 900 & 1000 \\
\hline
\end{tabular}

L'économiste a évalué les dégâts causés par chacune de ces crues (en millions de F) :

\begin{tabular}{|l|c|c|c|c|c|c|}
\hline Crue en $\mathbf{~ m}^{3} / \mathbf{s}$ & 400 & 500 & 600 & 700 & 800 & 900 \\
\hline Dégâts en MF & 40 & 70 & 125 & 170 & 170 & 210 \\
\hline
\end{tabular}

En tenant compte de la probabilité des crues, on peut calculer l'espérance mathématique des dégâts annualisés en fonction d'un débit de protection :

\begin{tabular}{|l|c|c|c|c|c|c|}
\hline $\begin{array}{l}\text { Débit de protection } \\
\text { en } \mathbf{~ m}^{3 / 5}\end{array}$ & 400 & 500 & 600 & 700 & 800 & 900 \\
\hline $\begin{array}{l}\text { Dégâts annualisés } \\
\text { en MF }\end{array}$ & 9 & 6 & 2,5 & 0,5 & 0,2 & 0,1 \\
\hline
\end{tabular}

Mais cette protection a un coût que l'on chiffre en valeurs annualisées :

\begin{tabular}{|l|c|c|c|c|c|c|}
\hline $\begin{array}{l}\text { Débit de protection } \\
\text { en } \mathbf{~ m}^{3 / \mathbf{s}}\end{array}$ & 400 & 500 & 600 & 700 & 800 & 900 \\
\hline $\begin{array}{l}\text { Coût annualisé des } \\
\text { ouvrages en MF }\end{array}$ & 0 & 0,5 & 1 & 1,6 & 2,2 & 3,2 \\
\hline
\end{tabular}


C'est ainsi que l'on peut construire un graphique simple résumant les résultats précédents en calculant, en valeurs annualisées, le bénéfice (la diminution des dégâts) moins le coût des ouvrages, cf. figure 1 .

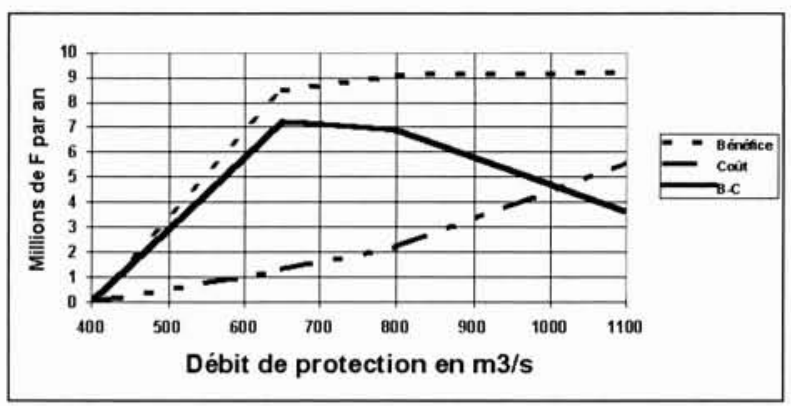

1. Bilan global tenant compte de la probabilité des crues.

Dans ce cas, l'optimum économique se situe vers $650 \mathrm{~m}^{3} / \mathrm{s}$; ce résultat dépend bien évidemment de la fonction de répartition des crues.

\section{II — LE RISQUE}

La notion de Temps de Retour est souvent mal comprise ; il est intéressant de calculer le taux de défaillance d'un ouvrage, par exemple une digue, pendant un certain nombre d'années en fonction de la protection qu'il apporte, exprimée en probabilité ou en Temps de Retour, que la valeur de protection ne soit pas dépassée. Le tableau 1 fournit ces résultats qui sont indépendants de toute loi de probabilité ; on suppose simplement que les événements sont indépendants d'une année à l'autre.

\section{III — L'ÉCHANTILLONNAGE}

Quelle que soit la méthode utilisée pour la prédétermination des crues, on va être amené à construire une fonction de répartition. Celle-ci sera établie, à partir de connaissances et à partir d'observations (crues, pluies extrêmes etc.) ; or, on ne possède que des échantillons réduits, souvent quelques

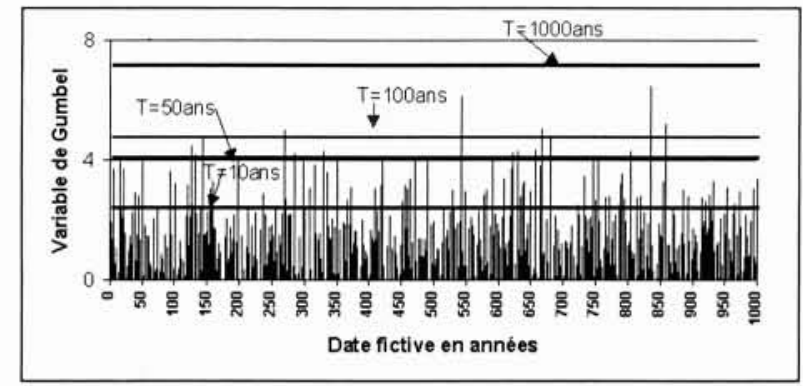

2. Simulation de $\mathbf{1 0 0 0}$ ans d'une variable de Gumbel.

dizaines d'années, d'où une incertitude d'échantillonnage sur le calcul de certains paramètres statistiques. La figure 2 correspond à une simulation aléatoire d'une variable de Gumbel (modèle courant de valeurs extrêmes) sur 1000 années fictives; on devine que selon la période courte (par exemple 50 ans) que l'on prend comme référence, on observe ou non des phénomènes différents, sans qu'il y ait là la moindre cause climatologique.

L'exemple suivant a été obtenu par simulation de 1000 années de pluies journalières maximales annuelles tirées d'une population semblable à l'échantillon historique des pluies à Coursegoules (près de Nice) ; on a pris, par simplicité, un modèle de Gumbel. Puis, on calcule, à partir de l'année 100 , et ceci, pour chaque année, la valeur centennale que l'on aurait obtenue en ne connaissant que les 50 années précédentes. On observe (figure 3 ) que cette évaluation fluctue,

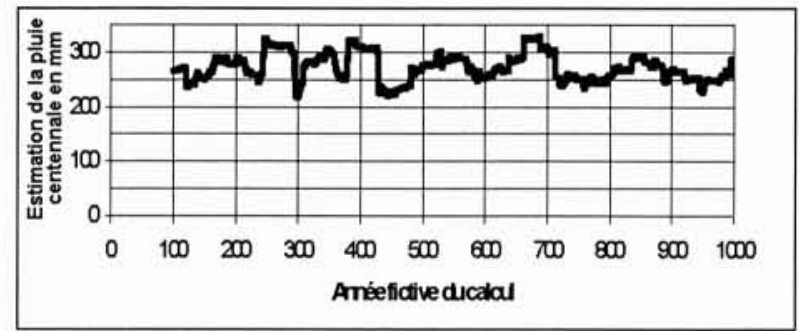

3. Estimation sur périodes glissantes de $\mathbf{5 0}$ ans de la pluie à partir d'une simulation de pluies maximales annuelles journalières gumbéliennes de moyenne $112 \mathrm{~mm}$ et d'écart type $50 \mathrm{~mm}$.

Tableau 1- Taux de défaillance en fonction de la protection et de la durée d'observation.

\begin{tabular}{|l|r|r|r|r|r|r|r|}
\hline $\begin{array}{l}\text { Protection contre les crues } \\
\text { en années de retour : }\end{array}$ & 2 & 10 & 20 & 50 & 100 & 200 & 1000 \\
\hline $\begin{array}{l}\text { Probabilité annuelle } \\
\text { au non dépassement: }\end{array}$ & 0,5 & 0,9 & 0,95 & 0,98 & 0,99 & 0,995 & 0,999 \\
\hline Défaillance en : & & & & & & & \\
1 an & $50 \%$ & $10 \%$ & $5 \%$ & $2 \%$ & $1 \%$ & $0,5 \%$ & $0,1 \%$ \\
2 ans & $75 \%$ & $19 \%$ & $10 \%$ & $4 \%$ & $2 \%$ & $1 \%$ & $0,2 \%$ \\
10 ans & $100 \%$ & $65 \%$ & $40 \%$ & $18 \%$ & $10 \%$ & $5 \%$ & $1 \%$ \\
20 ans & $100 \%$ & $88 \%$ & $64 \%$ & $33 \%$ & $18 \%$ & $10 \%$ & $2 \%$ \\
50 ans & $100 \%$ & $99 \%$ & $92 \%$ & $64 \%$ & $39 \%$ & $22 \%$ & $5 \%$ \\
100 ans & $100 \%$ & $100 \%$ & $99 \%$ & $87 \%$ & $63 \%$ & $39 \%$ & $10 \%$ \\
200 ans & $100 \%$ & $100 \%$ & $100 \%$ & $98 \%$ & $87 \%$ & $63 \%$ & $18 \%$ \\
1000 ans & $100 \%$ & $100 \%$ & $100 \%$ & $100 \%$ & $100 \%$ & $99 \%$ & $63 \%$ \\
\hline
\end{tabular}


mais que le résultat reste raisonnable, avec une fluctuation d'estimation de l'ordre de $20 \%$ dans la plupart des cas.

\section{IV — MÉTHODES DE PRÉDÉTERMINATION DES CRUES}

\subsection{Méthodes probabilistes sur les crues}

On peut être tenté d'ajuster directement les débits ou volumes de crues à des lois de probabilité plus ou moins complexes ; mais, bien souvent, comme le phénomène pluies-débits est complexe, rien ne prouve qu'un modèle ajusté sur les valeurs observées des crues puisse être extrapolable ; certains auteurs utilisent des méthodes de régionalisation pour tenter d'augmenter artificiellement la longueur des échantillons. Nous rappellerons ci-après une méthodologie qui a le grand avantage d'utiliser l'information hydrologique et l'information pluviométrique, la méthode du GRADEX et sa dérivée, la méthode AGREGEE.

\subsection{Notions d'une durée caractéristique de la réponse d'un bassin versant aux pluies}

Les méthodes suivantes vont travailler sur des volumes de pluies et des volumes de crues ; aussi est-il important de définir un pas de temps pour calculer ces volumes. En effet, pour une probabilité donnée, la pluie efficace qui donne le plus grand débit de pointe est celle qui correspond à la durée caractéristique de concentration du bassin versant. Pour illustrer cela, nous avons défini une fonction de transfert pluiedébit (un hydrogramme unitaire) de 12 heures et avons calculé les hydrogrammes causés respectivement par une pluie centennale de 1 heure, de 2 heures, jusqu'à 24 heures (valeurs prises à la station de Veynes près de Gap). On constate effectivement que le maximum est obtenu pour la pluie de durée correspondante à la durée de passage de l'essentiel en volume de l'hydrogramme unitaire ; on observe également que ce maximum est voisin pour des durées autour de celle du maximum.

\begin{tabular}{|l|c|c|c|c|c|c|c|}
\hline $\begin{array}{l}\text { Durée de la pluie } \\
\text { centennale }\end{array}$ & $1 \mathrm{~h}$ & $2 \mathrm{~h}$ & $4 \mathrm{~h}$ & $6 \mathrm{~h}$ & $12 \mathrm{~h}$ & $18 \mathrm{~h}$ & $24 \mathrm{~h}$ \\
\hline $\begin{array}{l}\text { Valeur de la pluie } \\
\text { en mm }\end{array}$ & 42 & 53 & 66 & 76 & 95 & 115 & 120 \\
\hline
\end{tabular}

\begin{tabular}{|c|c|c|c|c|c|c|c|}
\hline Temps en heure & 0 & 1 & 2 & 3 & 4 & 5 & 6 \\
\hline $\begin{array}{l}\text { Hydrogramme } \\
\text { unitaire }\end{array}$ & 0 & 4,1 & 10,5 & 16,4 & 18,2 & 16,4 & 12,7 \\
\hline Temps en heure & 7 & 8 & 9 & 10 & 11 & 12 & \\
\hline $\begin{array}{l}\text { Hydrogramme } \\
\text { unitaire }\end{array}$ & 9,1 & 6,4 & 3,6 & 1,8 & 0,9 & 0 & \\
\hline
\end{tabular}

L'essentiel du volume ( $80 \%$ ) passe en 6 heures ou encore, 6 pas de temps dépassent en valeurs la moitié du débit de pointe. Les pointes observées sont les suivantes :

\begin{tabular}{|l|c|c|c|c|c|c|c|}
\hline $\begin{array}{l}\text { Durée de la pluie de } \\
\text { référence en heures }\end{array}$ & 1 & 2 & 4 & 6 & 12 & 18 & 24 \\
\hline $\begin{array}{l}\text { Max. débit observé } \\
\text { en mm }\end{array}$ & 7,6 & 9,2 & 10,5 & 10,5 & 7,9 & 6,4 & 5 \\
\hline
\end{tabular}

C'est ainsi qu'EdF préconise de prendre comme durée caractéristique la moyenne ou médiane des durées pendant lesquelles passent $80 \%$ du volume de crue, le CEMAGREF la durée pendant laquelle le débit est supérieur à la moitié du débit de pointe.

\subsection{Hypothèses fortes de la méthode du gradex}

\subsubsection{Sur les pluies extrêmes}

La fréquence au non-dépassement des précipitations extrêmes annuelles, locales ou spatiales (de quelques $\mathrm{km}^{2}$ à plusieurs milliers de $\mathrm{km}^{2}$, de durée une heure à quelques jours, est à décroissance exponentielle simple : $\log (1-\mathrm{F}$ (Pluie de durée $D)$ ) $=-$ Pluie $(D) / a+$ cste où $a$ est appelé gradex (gradient de l'exponentielle) des pluies de durée $D$. L'estimation de ce paramètre, essentiel pour la méthode, car c'est le paramètre qui donne l'accroissement de la variable en fonction du temps de retour, se fait en général en considérant des saisons et en utilisant plus d'information que les maxima saisonniers.

\subsubsection{Hypothèses sur la rétention du bassin}

On appelle rétention pour une crue la différence entre la lame d'eau tombée et le volume écoulé lors de la crue (une fois enlevé le débit de base). Cette quantité est variable d'une crue à l'autre et dépend des conditions d'humidité du sol et de la précipitation. On fait l'hypothèse que la distribution probabiliste de cette rétention tend à être la. même lorsque l'épisode de pluie devient important ; certains auteurs expriment cette hypothèse de façon plus simple, mais peut-être moins générale : lorsque l'épisode de pluie est important, tout accroissement de pluie s'écoule, c'est-à-dire que le coefficient d'écoulement de la pluie supplémentaire tend vers 1 (mais pas celui de l'épisode total). Cela n'est évidemment pas valable pour tous les bassins (par exemple les bassins volcaniques très perméables).

On peut alors montrer que la fonction de répartition des volumes de crues définis sur la durée $D$ tend asymptotiquement vers une parallèle (sur un papier de Gumbel qui linéarise la distribution exponentielle des pluies maximales de durée $D$ ) à la droite des pluies de durée $D$, en prenant évidemment la même unité pour les pluies et les volumes. Cette

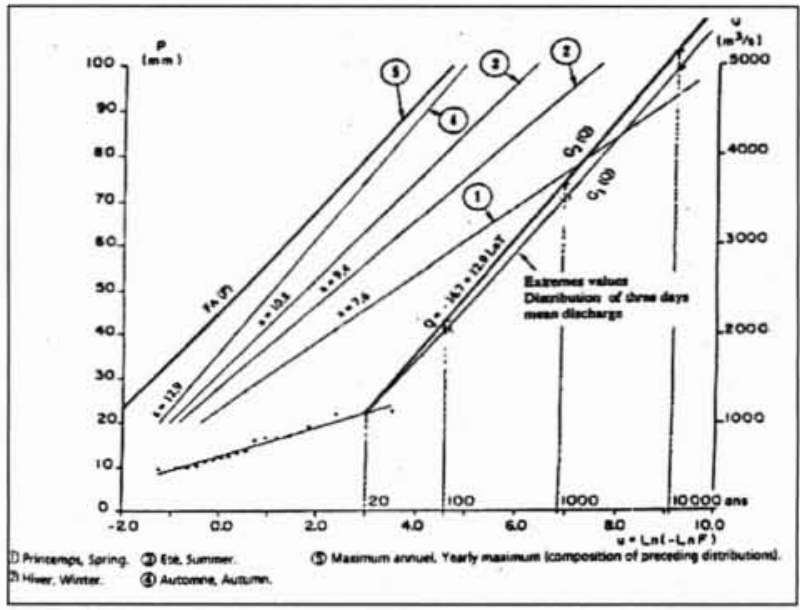

4. Application du GRADEX aux débits de 72 heures de l'Allier au Veurdre. 
extrapolation des volumes est faite à partir d'un temps de retour estimé selon le bassin, souvent de l'ordre de 10 à 20 ans ; si l'on change ce point dit point pivot, l'écart des résultats est faible, en valeur relative pour des temps de retour élevés, cf. figure 4 tirée de [1].

\subsection{Passage des volumes sur la durée $D$ aux pointes de crues}

Le rapport Pointe/Débit moyen sur la durée $D$ est appelé coefficient de forme ; ce coefficient varie évidemment d'une crue à l'autre et dépend de $D$ (si $D$ augmente, le coefficient augmente). On obtient ce coefficient à partir des crues observées et on examine s'il est fonction de l'importance de la crue. Si la durée $D$ est bien choisie, il est de l'ordre de 1,5.

On comprend que le choix de cette durée $D$ n'a pas besoin d'être fait de manière très précise ; si $D$ est choisi un peu grand, l'augmentation des pluies en fonction du temps de retour sera plus faible en valeur relative, mais le coefficient de forme sera plus grand qu'avec une durée $D$ plus courte. Dans la pratique, on détermine approximativement cette durée $D$ et on refait les calculs pour des durées $D$ moitié et double.

\subsubsection{Conclusions sur la méthode du GRADEX}

L'énorme intérêt de la méthode du GRADEX est de tenir compte de la distribution des pluies extrêmes de durées voisines de celle du temps de concentration du bassin pour extrapoler les volumes de crue sur $D$. En effet, les précipitations sont plus faciles à mesurer que les fortes crues et sont souvent connues depuis plus longtemps et plus régionalisables.

\section{V — PROLONGEMENTS DE LA MÉTHODE DU GRADEX}

L'objectif de la méthode du GRADEX est d'obtenir une estimation des crues de fréquence rare de type $10^{-3}$ à $10^{-6}$ au dépassement. La méthode AGREGEE a pour but de s'intéresser aux crues plus fréquentes du type $10^{-2}$ à $10^{-3}$. Schématiquement, au lieu d'extrapoler la fonction de répartition des volumes de crues sur $d$ parallèlement à la droite des pluies extrêmes sur $D$ à partir d'un temps de retour de 10 ou 20 ans,

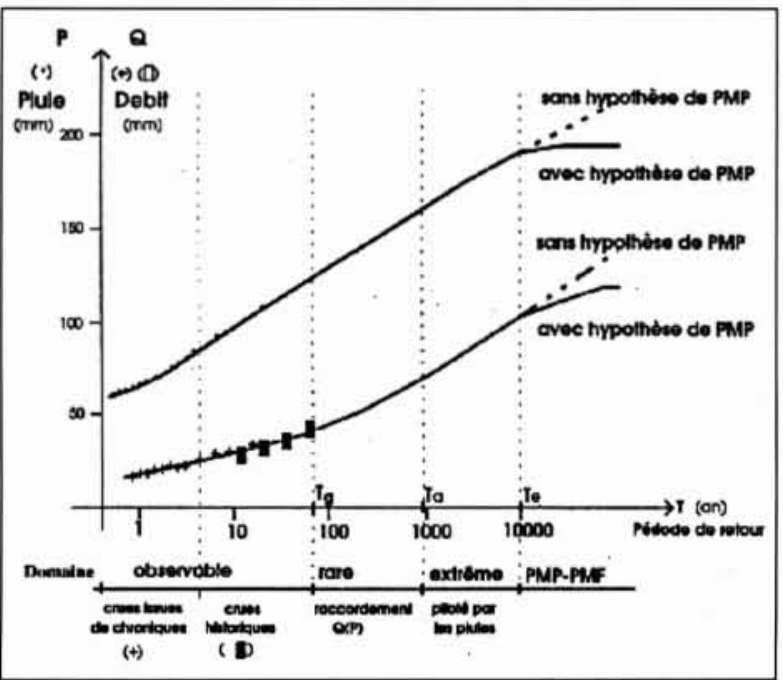

5. Les domaines du modèle AGREGEE. on lisse par un modèle le passage de la fonction de répartition des crues observées à la parallèle à la loi des pluies par une fonction. "Un des points forts du modèle est une bonne formulation de la progressivité de la distribution des volumes moyens reliant de façon continue trois domaines de fonctionnement différents des crues"

- le domaine observable avec éventuellement la prise en compte de l'information historique,

- le domaine rare où les données sur les pluies relayent progressivement celles concernant les débits,

- et le domaine extrême lié au comportement asymptotique de la loi des crues à celle des pluies."

La figure 5, fournie par Michel Lang, illustre les résultats de cette méthode.

\section{VI $\square$ CONCLUSIONS}

Il ne faut pas cacher qu'il existe à l'heure actuelle plusieurs débats :

- sur l'intérêt des méthodes maximalistes dites Pluie Maximale Probable PMP, Crue Maximale Probable,

- sur la validité des lois de probabilité des débits extrêmes ou des pluies extrêmes.

Ces débats ne sont pas prêts de s'arrêter, faute de possibilité de vérification. L'intérêt des méthodes probabilistes du type GRADEX est qu'elles font appel à des hypothèses simples et ne retiennent que l'essentiel du phénomène crue. Elles ont été appliquées sur de très nombreux bassins de différents pays.

\section{BIBLIOGRAPHIE SUCCINCTE}

[1] Bouvard M., Garros Berthet et al. - Les crues de projet des barrages : Méthode du GRADEX. Bulletin du comité français des grands

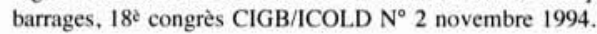

Note : c'est un des documents les plus explicites sur la méthode du GRADEX; il comprend également la description d'autres méthodes ainsi que de nombreux exemples.

[2] Groupe de travail du CFGB-Détermination des crues de projet. CIGB $11^{\circ}$ congrès Q41R8 Madrid 1973.

[3] Duband D., Garçon R. - Extreme precipitation values and their frequencies. Can we rely on our estimates. CIGB Grenade 1992.

[4] Guil.ot P., Duband D. - La méthode du GRADEX pour le calcul de la probabilité des crues à partir des pluies. IASH Publication $N^{\circ} 84,1967$.

[5] LANG M., OberLin G.- Preliminary test for mapping the 100 year flood with the AGREGEE model. Technische Universitat Braunschweig FRIEND conference 11-15 October 1993 Germany.

[6] Margoum M. - Estimations des crues rares et extrêmes : le modèle AGREGEE. Conception et premières validations. Thèse, CEMAGREF et Ecole des Mines de Paris, juillet 1992.

[7] Miquel. J. - Guide pratique d'estimation des probabilités de crues. Eyrolles 1984. 